\title{
ON THE NUMBER OF VARIETIES OF GROUPS
}

\author{
L. G. KOVACS \\ (Received 18 January 1967)
}

There are infinitely, but at most continuously, many varieties of groups; the precise cardinal is unknown. It is easy to see that if there is no infinite properly descending chain of varieties (equivalently, if the laws of every variety have a finite basis), then the number of varieties is countable infinity; the converse implication does not seem to have been proved. This note presents an argument which implies that if the locally finite or the locally nilpotent varieties fail to satisfy the minimum condition, then there are continuously many such varieties. Alternatively, one can conclude that if a locally finite or locally nilpotent variety $\mathfrak{B}$ has a finite basis for its laws but some subvariety $\mathfrak{U}$ of $\mathfrak{B}$ has none, then there are continuously many varieties between $\mathfrak{U}$ and $\mathfrak{B}$. This points again to the interesting question: is every locally finite [locally nilpotent] variety $\mathfrak{U}$ contained in a suitable locally finite [locally nilpotent] variety $\mathfrak{B}$ which has a finite basis for its laws? (That is, must $\mathfrak{U}^{(n)}$ be locally finite [locally nilpotent] for some finite $n$ ?) For, if the answer were affirmative, it would follow that the number of locally finite [locally nilpotent] varieties is either countable or the cardinal of the continuum, depending exactly on the existence of finite bases for the laws of such varieties. For soluble locally finite [soluble locally nilpotent] varieties the answer is affirmative (since soluble groups of finite exponent are locally finite [soluble Engel groups are locally nilpotent; cf. K. W. Gruenberg, Illinois J. Math. 3 (1951), 151-167]), and so for these varieties the above alternative is now established.

For notation, terminology, and standard results, the reader is referred to Hanna Neumann's book [Varieties of groups, Springer, Berlin etc., 1967].

THEOREM. Suppose that there exists an infinite properly descending chain

$$
\mathfrak{B}_{\mathbf{1}}>\mathfrak{B}_{\mathbf{2}}>\cdots>\mathfrak{B}_{j}>\cdots
$$

of varieties such that, for every positive integer $i$, the subvarieties of the variety $\operatorname{var} F_{i}\left(\mathfrak{B}_{1}\right)$ [generated by the free group $F_{i}\left(\mathfrak{B}_{1}\right)$ of rank $i$ in $\mathfrak{B}_{1}$ ] satisfy the minimum condition. Then $\mathfrak{B}_{1}$ has continuously many subvarieties which contain $\bigcap_{j} \mathfrak{B}_{j}$. 
Proof. Put $i \mathfrak{B}_{j}=\operatorname{var} F_{i}\left(\mathfrak{B}_{j}\right)$ for every pair of positive integers $i, j$; note that ${ }^{i B_{j}}$ is increasing in $i$ and decreasing in $j$. For each function $f: k \rightarrow f(k)$ from the set $I$ of positive integers to the set $\{0,1\}$, define a variety $\mathfrak{B}_{f}$ between $\mathfrak{B}_{1}$ and $\cap\left\{\mathfrak{B}_{j}: j \in I\right\}$ by

$$
\mathfrak{B}_{f}=\cup\left\{a(k) \mathfrak{B}_{b(k+f(k))}: k \in I\right\},
$$

where $a, b$ are suitable strictly increasing functions from $I$ to $I$ : the task is to choose $a$ and $b$ so that $\mathfrak{V}_{f} \neq \mathfrak{B}_{g}$ if $f \neq g$. In fact, $a$ and $b$ will be defined so that if $m$ is the first integer on which $f$ and $g$ disagree, then

$$
\mathfrak{B}_{f}(F) \neq \mathfrak{B}_{\mathfrak{g}}(F)
$$

where $F$ is any (absolutely) free group of rank $a(m)$.

Note that

for,

$$
\mathfrak{B}_{f}(F)=\cap\left\{{ }^{a(k)} \mathfrak{B}_{b(k+f(k))}(F): k \leqq m\right\}:
$$

$$
\mathfrak{B}_{f}(F)=\cap\left\{a^{a(k)} \mathfrak{B}_{b(k+f(k))}(F): k \in I\right\}
$$

by definition, while, for $k>m$,

$$
a(k) \mathfrak{B}_{b(k+f(k))} \leqq \mathfrak{B}_{b(m+f(m))}
$$

so that, since the rank of $F$ is $a(m)$,

$$
{ }^{a(k)} \mathfrak{B}_{b(k+f(k))}(F) \geqq \mathfrak{B}_{b(m+f(m))}(F)={ }^{a(m)} \mathfrak{B}_{b(m+f(m))}(F) .
$$

A similar expression holds for $\mathfrak{B}_{g}(F)$. As

$$
\cup\left\{a(k) \mathfrak{B}_{b(k+f(k))}: k \leqq m\right\}
$$

and the variety defined correspondingly with $g$ in place of $f$ are generated by their $a(m)$-generator groups, $(*)$ is equivalent to

$$
\cup\left\{a^{a(k)} \mathfrak{B}_{b(k+f(k))}: k \leqq m\right\} \neq \cup\left\{a(k) \mathfrak{B}_{b(k+g(k))}: k \leqq m\right\} .
$$

Let $f(m)=0, g(m)=1$, say. Because of the minimal choice of $m$, the left hand side of $\left({ }^{* *}\right)$ contains the right hand side, and so $\left(^{* *}\right)$ is equivalent to the denial of the converse inclusion. Since the left hand side contains ${ }^{a(m)} \mathfrak{B}_{b(m)}$ while the right hand side is contained in

$$
{ }^{a(1)} \mathfrak{B}_{b(2)} \text { or } \quad{ }^{a(m-1)} \mathfrak{B}_{1} \cup^{a(m) \mathfrak{B}_{b(m+1)}} \text { as } m=1 \text { or } m>1 \text {, }
$$

it suffices to choose $a$ and $b$ so that

$$
{ }^{a(1)} \mathfrak{B}_{b(1)} \neq{ }^{a(1)} \mathfrak{B}_{b(2)}
$$

and

$$
a(m) \mathfrak{B}_{b(m)}{ }^{a(m-1)} \mathfrak{B}_{1} \cup^{a(m)} \mathfrak{B}_{b(m+1)}
$$

whenever $1<m \in I$. 
Now $\cup\left\{\mathfrak{B}_{j}: i \in I\right\}=\mathfrak{B}_{j}$ and $\mathfrak{B}_{1}>\mathfrak{B}_{2}$ ensure that ${ }^{i \mathfrak{B}_{1}}={ }^{i \mathfrak{B}_{2}}$ cannot hold for every $i$ in $I$. Let $a(1)$ be the first value of $i$ for which ${ }^{i \mathfrak{B}_{1}}>{ }^{i \mathfrak{B}_{2}}$, and let $b(\mathbf{1})=\mathbf{1}$ : then $b(2)>b(1)$ suffices for the validity of $(1)$. To complete the definition of $a$ and $b$ inductively, suppose that

$$
a(1), \cdots, a(m-1), b(1), \cdots, b(m-1)
$$

have already been defined, and that $(1), \cdots,(m-1)$ are valid whenever $b(m)$ is chosen greater than $b(m-1)$. Consider the descending chain

$$
a(m-1) \mathfrak{B}_{1} \cap \mathfrak{B}_{b(m-1)+j}
$$

of subvarieties of ${ }^{a(m-1)} \mathfrak{B}_{1}$. By hypothesis, this must be constant beyond the first few terms: thus one can choose $b(m)$ so that $b(m)>b(m-1)$ and

$$
{ }^{a(m-1)} \mathfrak{B}_{1} \cap \mathfrak{B}_{b(m)}={ }^{a(m-1)} \mathfrak{B}_{1} \cap \mathfrak{B}_{b(m)+1} .
$$

By the modular law,

would now imply

$$
\mathfrak{B}_{b(m)} \leqq{ }^{a(m-1)} \mathfrak{B}_{1} \cup \mathfrak{B}_{b(m)+1}
$$

$$
\mathfrak{B}_{b(m)}=\left({ }^{a(m-1)} \mathfrak{B}_{1} \cap \mathfrak{B}_{b(m)}\right) \cup \mathfrak{B}_{b(m)+1}=\mathfrak{B}_{b(m)+1},
$$

contrary to the fact that $\mathfrak{B}_{b(m)}>\mathfrak{B}_{b(m)+1}$ : thus

$$
\mathfrak{B}_{b(m)}{ }^{a(m-1)} \mathfrak{B}_{1} \cup \mathfrak{B}_{b(m)+1} \text {. }
$$

Since $\mathfrak{B}_{b(m)}=\cup\left\{\mathfrak{i \mathfrak { B }}_{b(m)}: i \in I\right\}$, for sufficiently large choice of $a(m)$ one must obtain

But

$$
{ }^{a(m)} \mathfrak{B}_{b(m)} \neq{ }^{a(m-1) \mathfrak{B}_{1} \cup \mathfrak{B}_{b(m)+1}} .
$$

$$
{ }^{a(m)} \mathfrak{B}_{b(m+1)} \leqq{ }^{a(m)} \mathfrak{B}_{b(m)+1} \leqq \mathfrak{B}_{b(m)+1}
$$

whenever $b(m+1)>b(m)$, and so $(m)$ is valid for every such choice of $b(m+1)$. The inductive step, and with it the definition of $a$ and $b$, is now complete.

Australian National University

Canberra 